\title{
How Reactivity Variability of Biofunctionalized Particles Is Determined by Superpositional Heterogeneities
}

\author{
Rafiq M. Lubken ${ }^{1,3}$, Arthur M. de Jong ${ }^{2,3}$ \& Menno W.J. Prins ${ }^{1,2,3, *}$
}

\begin{abstract}
${ }^{1}$ Department of Biomedical Engineering, Eindhoven University of Technology, Eindhoven, 5612 AP, the Netherlands.

${ }^{2}$ Department of Applied Physics, Eindhoven University of Technology, Eindhoven, 5612 AP, the Netherlands.

${ }^{3}$ Institute for Complex Molecular Systems (ICMS), Eindhoven University of Technology, Eindhoven, 5612 AP, the Netherlands.
\end{abstract}

*email: m.w.j.prins@tue.nl

\section{SUPPLEMENTARY NOTES}

\section{Quantitative points accumulation in nanoscale topography (qPAINT)}

\section{1 qPAINT measurement}

Quantitative points accumulation in nanoscale topography (qPAINT) is a fluorescence-based measurement technique by which quantitative information on the number of molecules on complexes or surfaces can be obtained. ${ }^{1}$ The method exploits the well-defined and controllable binding behavior of dye-labeled single-stranded DNA (ssDNA) imager strands to ssDNA docking strands (see Figure S1), which are referred to in this paper as targeting moieties. The number of targeting moieties can be determined via imager strands that transiently bind to the complex- or surface-bound ssDNA docking strands, causing observable binding and unbinding events (see Figure S1a). The distribution of the observed unbound times (i.e. dark times) of imager strands to ssDNA docking strands in a region of interest (ROI), depends on the number of ssDNA docking strands in this ROI for a given imager strand concentration (see Figure S1b). Under the assumption that no simultaneous binding events occur in a single $\mathrm{ROI}$ at each given time point, the number of targeting moieties per $\mathrm{ROI}$ can be calculated using:

$$
N_{\text {moiety }}=\frac{1}{k_{\mathrm{on}} c_{i} \tau_{d}}
$$

with $N_{\text {moiety }}$ the number of targeting moieties in the ROI (in this paper a single particle), $k_{\text {on }}$ the association rate constant of the interaction between the imager strand and the docking strand, $c_{i}$ the imager strand concentration, and $\tau_{d}$ the mean observed dark time. Since $k_{\text {on }}$ is a molecularly determined constant, ${ }^{1,2}$ and $c_{i}$ an experimental condition, the observable $\tau_{d}$ reveals the number of targeting moieties per particle. The precision with which $N_{\text {moiety }}$ can be determined experimentally depends on the number of observed dark states corresponding to Poisson statistics (see Equation 5).
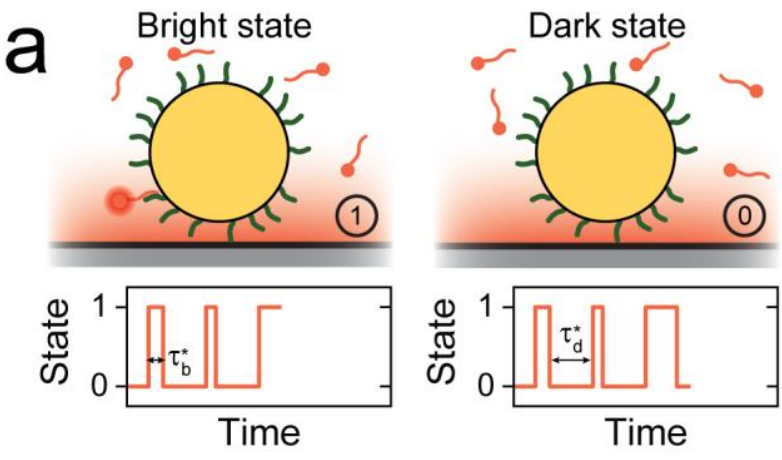

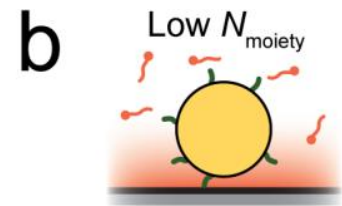

High $N$
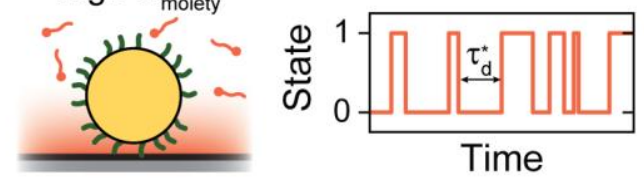

Figure S1. Measurement principle of quantitative points accumulation in nanoscale topography (qPAINT) to quantify the number of targeting moieties on a single particle. (a) The binding and unbinding behavior of dye-labeled ssDNA imager 
strands (red) to ssDNA docking strands (green) yields observable bright states (state 1) with length $\tau_{b}^{*}$ and dark states (state 0 ) with length $\tau_{d}^{*}$. Only the associated ssDNA imager strands close to the glass substrate yield a fluorescent signal due to total internal reflection excitation, causing an evanescent field (red gradient). (b) For particles with a low number of targeting moieties $N_{\text {moiety }}$, the observed dark times are on average longer than for particles with a high number of targeting moieties; using the mean observed dark time $\tau_{d}$, the number of targeting moieties can be determined using Equation $\mathrm{S} 1$.

\subsection{Analysis of qPAINT data}

In Figure S2 the data analysis procedure of experimental qPAINT data is shown. Figure S2a visualizes an example of the integrated intensity (blue) of a single ROI (i.e. a single particle) as a function of time. The red dashed line indicates a threshold of $\mu_{b}+5 \sigma_{b}$ with $\mu_{b}$ and $\sigma_{b}$ the mean and the standard deviation of the background signal respectively. Using this threshold, the bright and dark times can be extracted from the integrated intensity trace. In order to correct for intensity fluctuations in the bright time and blinking, which might lead to falsely detected binding and unbinding events, two filters were used. The first filter removes single frames below the intensity threshold with neighboring frames above the intensity threshold; these events are regarded as false unbinding events and set to a bound state, under the condition that the integrated intensity in this particular frame is above $\mu_{b}+\sigma_{b}$. The second filter removes single frames above the intensity threshold with neighboring frames below the intensity threshold; these are regarded as false binding events and set to an unbound state, under the conditions that the integrated intensity in this particular frame is below $\mu_{b}+9 \sigma_{b}$. The red solid line (see inset) visualizes the state trace that results from thresholding the integrated intensity profile; a high level indicates a bound state from which the bright time was extracted, and a low level indicates an unbound state from which the dark time was extracted.

Figure S2b shows two cumulative distribution functions (CDFs), and two histograms of the mean dark and bright times of all observed lifetimes on a single particle. For the CDF of the dark times, a singleexponential distribution (blue dashed line) with $\tau_{d}=12.2 \pm 0.1 \mathrm{~s}$ was observed indicating for a singlemolecular binding process. In contrast, the CDF for the bright times only exhibit an approximate singleexponential distribution (red dashed line) with $\tau_{b}=830 \pm 29 \mathrm{~ms}$ for $70 \%$ of all observed bright times. This effect might be explained by filtering the dark times with a length that equals a single frame; this results in a tail of merged, and thus longer, bright times. However, since $70 \%$ of the observed bright times and all observed dark times follow a single-exponential distribution, we neglected the influence of this effect on the quantitation of the number of targeting moieties per particle.
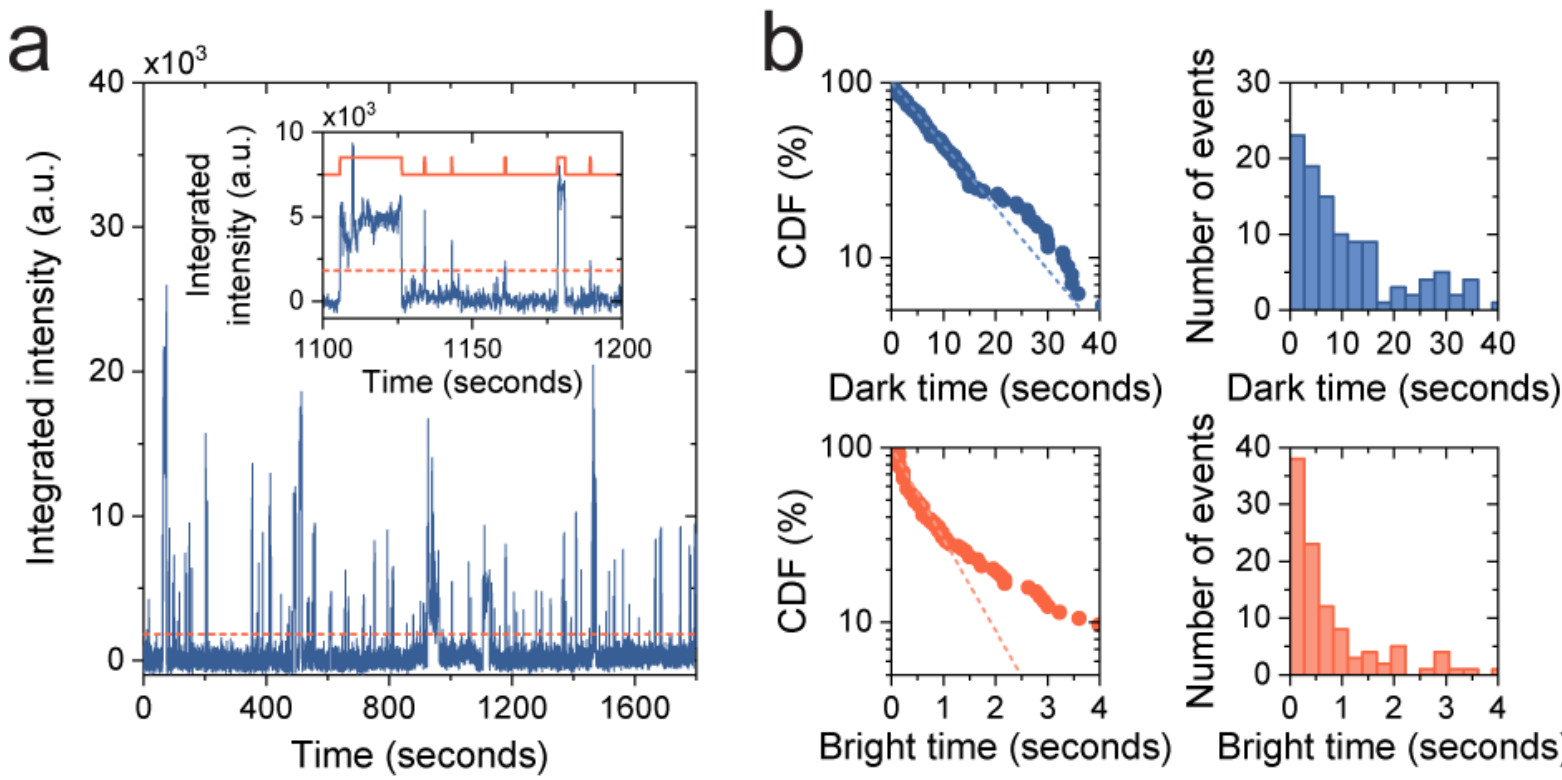

Figure S2. Data analysis procedure of experimental qPAINT data. (a) Integrated intensity in a region of interest (i.e. a single particle) as a function of time. The threshold (red dashed line) is determined by means of the background signal and has the value of $\mu_{b}+5 \sigma_{b}$ with $\mu_{b}$ and $\sigma_{b}$ the mean and the standard deviation of the background signal respectively. The inset shows a zoom-in in which individual bright and dark times can be observed after thresholding the integrated intensity (red solid line). (b) Lifetime analysis of the dark and bright times observed in a single particle. The cumulative distribution function (CDF) of all 
observed dark times shows a single-exponential distribution (blue dashed line) with $\tau_{d}=12.2 \pm 0.1 \mathrm{~s}$. The CDF of all observed bright times shows a single-exponential distribution (red dashed line) for approximately $70 \%$ of the observed bright times with $\tau_{b}=830 \pm 29 \mathrm{~ms}$. The errors indicated in the caption are fitting errors.

\subsection{Mass transport limitation in qPAINT experiments}

Since the number of targeting moieties per particle is calculated from the mean dark time $\tau_{d}$ per particle, no more than a single event should occur at a point in time per particle. If this condition is not met, the number of targeting moieties per particle is underestimated. ${ }^{3}$ However, in order to meet this condition, particles with a large number of targeting moieties (in this paper $\sim 400,000$ moieties per particle) pose an intrinsic imager strand transport problem since low imager strand concentrations are required $(\sim \mathrm{fM})$ in order to determine the number of targeting moieties per particle. Conventional qPAINT measurements $^{1-3}$ are performed at imager strand concentrations of $>100 \mathrm{pM}$ which result in a mean intermolecular distance $d_{\text {mol }}$ in solution of $<3 \mu \mathrm{m}$ with a corresponding characteristic diffusion time $\tau_{\text {diff }}$ of $<0.03 \mathrm{~s}$ where $\tau_{\text {diff }}$ is defined as the time required for the imager strand to diffuse over distance $d_{\mathrm{mol}}$ :

$$
\tau_{\text {diff }}=\frac{d_{\mathrm{mol}}^{2}}{D_{0}}
$$

with $d_{\text {mol }}$ the mean intermolecular distance where $d_{\mathrm{mol}} \cong \frac{1 \cdot 18 \cdot 10^{-9}}{\sqrt[3]{c_{i}}}$, and $D_{0}$ the diffusion coefficient of the imager strand. Under the assumption that $\tau_{\text {diff }} \ll \tau_{d}, \tau_{d}$ is indeed inversely proportional to $N_{\text {moiety }}$ and $c_{i}$ according to Equation S1. However, when low imager strand concentrations (sub-pM range) are used, the assumption that $\tau_{\text {diff }} \ll \tau_{d}$ does no longer hold; the observed association kinetics are then mass transport limited and the number of docking strands is underestimated.

In Figure S3 the results of a qPAINT experiment are given in which the mass transport limitation is clearly visible. Figure S3a shows the dependency of the number of active targeting moieties per particle, quantified by qPAINT, as a function of the ssDNA to particle ratio present in solution during incubation (blue). The top $x$-axis indicates the incubated ssDNA concentration and the $y$-axis the measured number of ssDNA targeting moieties per particle. For an increasing ratio, a linearly increasing number of targeting moieties per particle was observed at low ssDNA concentrations. This linear relation is similar to the linear relation presented in Figure $2 a$ (grey dashed line). However, at high ssDNA concentrations, the experimental data exhibit a deviating behavior from this linear dependency; in this regime, the imager strand concentration is in the low pM concentration range, and thus mass transport limits the observed transient binding behavior.

In Figure S3b the experimentally determined number of targeting moieties on a single particle and the characteristic diffusion time is given as a function of imager strand concentration. On the left, two samples, with an incubated ssDNA concentration of $333 \mathrm{nM}$ (dark blue) and $56 \mathrm{nM}$ (light blue), were measured each with three imager strand concentrations. Since no imager strand dependency exists, the conditions of $\tau_{\text {diff }} \ll \tau_{d}$ is met at lower ssDNA concentrations. However, on the right, the characteristic diffusion time is calculated as a function of imager strand concentration. Since mass transport effects start to appear at a ssDNA concentration of $667 \mathrm{nM}$ (see Figure S3a), with a corresponding imager concentration of $4 \mathrm{pM}$, the grey solid line indicates the threshold below which the condition of $\tau_{\text {diff }} \ll \tau_{d}$ is met. 


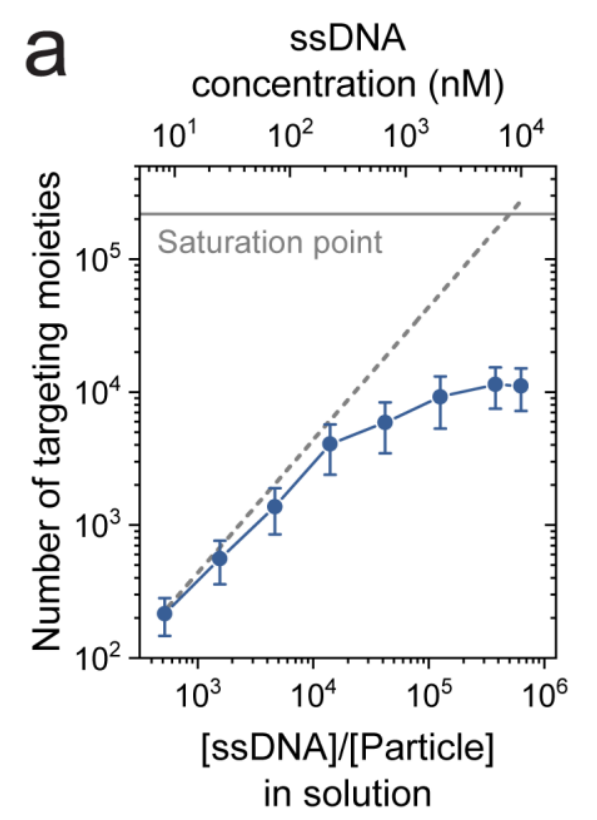

b
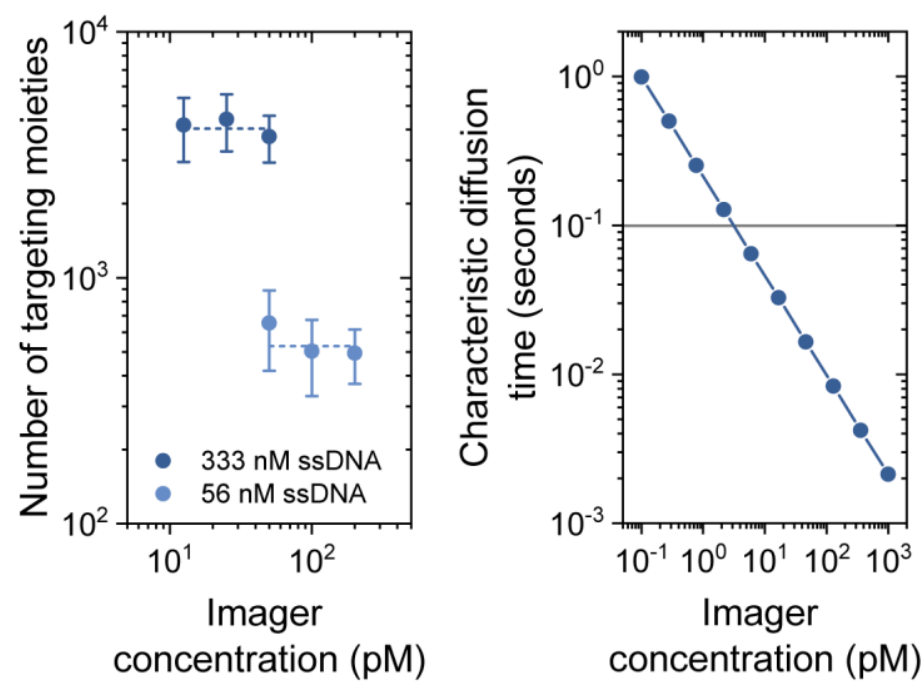

Figure S3. Mass transport limitation in the quantification of the number of targeting moieties per particle. (a) The number of targeting moieties (blue) as a function of the ssDNA to particle ratio in solution. The saturation point (grey solid line) of (2.0 \pm $0.2) \cdot 10^{5}$ targeting moieties per particle is determined by a supernatant assay (see Supplementary Note 3 ). The grey dashed line indicates the linear relation between the number of targeting moieties per particle present in solution and the number of observed targeting moieties presented in Figure $2 a$. The secondary $x$-axis reveals the incubated ssDNA concentration. The errors indicated in the panel are the standard deviations. (b) Left: two samples ( $333 \mathrm{~nm}$ and $56 \mathrm{nM}$ ssDNA) which were measured thrice with a different imager strand concentration. Right: calculated characteristic diffusion time as a function of imager strand concentration. The grey line indicates the threshold above which mass transport effects are visible in the quantitation of the number targeting moieties.

To solve this mass transport problem, only a fraction of the ssDNA molecules on the particle $(2.9 \%$ in this paper) has a sequence complementary to the used imager strand, which results in imager strand concentrations of $>5 \mathrm{pM}$. The remainder of the ssDNA molecules have a random, noncomplementary sequence with an equal length. Therefore, the $y$-axis of Figure 2a shows the measured number of ssDNA molecules after correcting from $2.9 \%$ to $100 \%$. 


\section{DNA points accumulation in nanoscale topography (DNA-PAINT)}

\subsection{DNA-PAINT measurements}

DNA points accumulation in nanoscale topography (DNA-PAINT) is a fluorescence-based measurement technique from which the spatial distribution of molecules on complexes or surfaces can be obtained. Similar to qPAINT, the well-defined and controllable binding behavior of dye-labeled imager strands to ssDNA docking strands is exploited. ${ }^{4}$ Hybridization of an imager strand to the ssDNA docking strand results in a fluorescent signal, from which the super-resolved location can be extracted using its diffraction-limited spot (see Figure S4). Therefore DNA-PAINT can be used to map the active targeting moieties on the surface of nanomaterials.

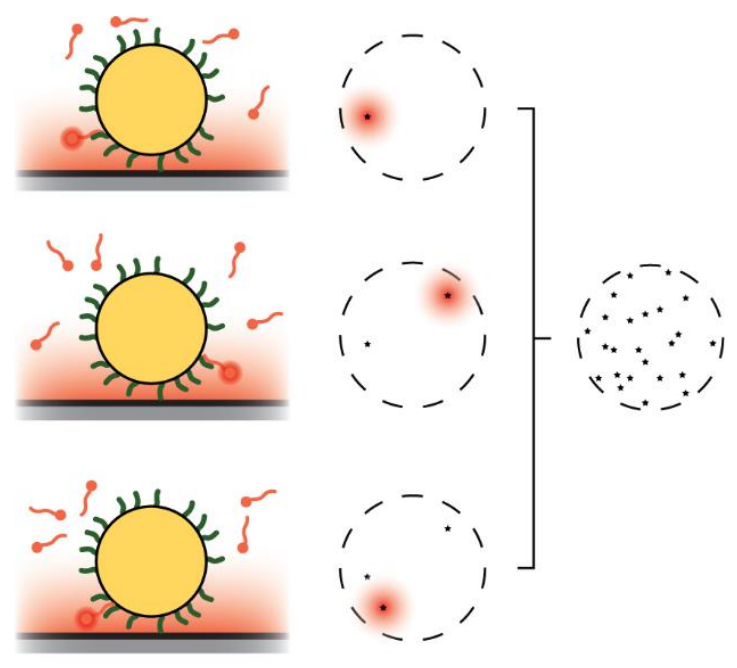

Figure S4. Measurement principle of DNA points accumulation in nanoscale topography (DNA-PAINT) to map the positions of targeting moieties on a single particle. The binding of dye-labeled SsDNA image strands (red) to ssDNA docking strands (green) yields an observable fluorescence signal. Only the associated ssDNA imager strands close to the glass substrate yield a fluorescent signal due to total internal reflection excitation, causing an evanescent field (red gradient). Since it concerns an isolated emitter, its diffractionlimited spot can be fitted with a point-spread function, from which the super-resolved position of the emitter can be determined (black star).

\subsection{D DNA-PAINT measurements}

In Figure S5, the results of a 3D DNA-PAINT measurement are shown. Here an astigmatic lens was used in order to obtain the z-position of the targeting moieties. Figure S5a shows the 3D positions of the targeting moieties on a single particle; the shape of the lower hemisphere of the particle can be distinguished. The z-coordinates span from approximately $0-1200 \mathrm{~nm}$ which suggests that the whole particle is imaged. However, the localization uncertainty in the z-direction is large compared to the uncertainty in the xy-direction, thus the targeting moiety positions were projected on the $x y$-plane in Figure S5b. From panels $a$ and $b$ can be concluded that the localized targeting moieties are mainly located on the lower hemisphere of the particle, and no targeting moieties are found on the top hemisphere of the particle. Therefore, it can be concluded that at least the lower hemisphere of the silica particles is imaged in a DNA-PAINT experiment. 

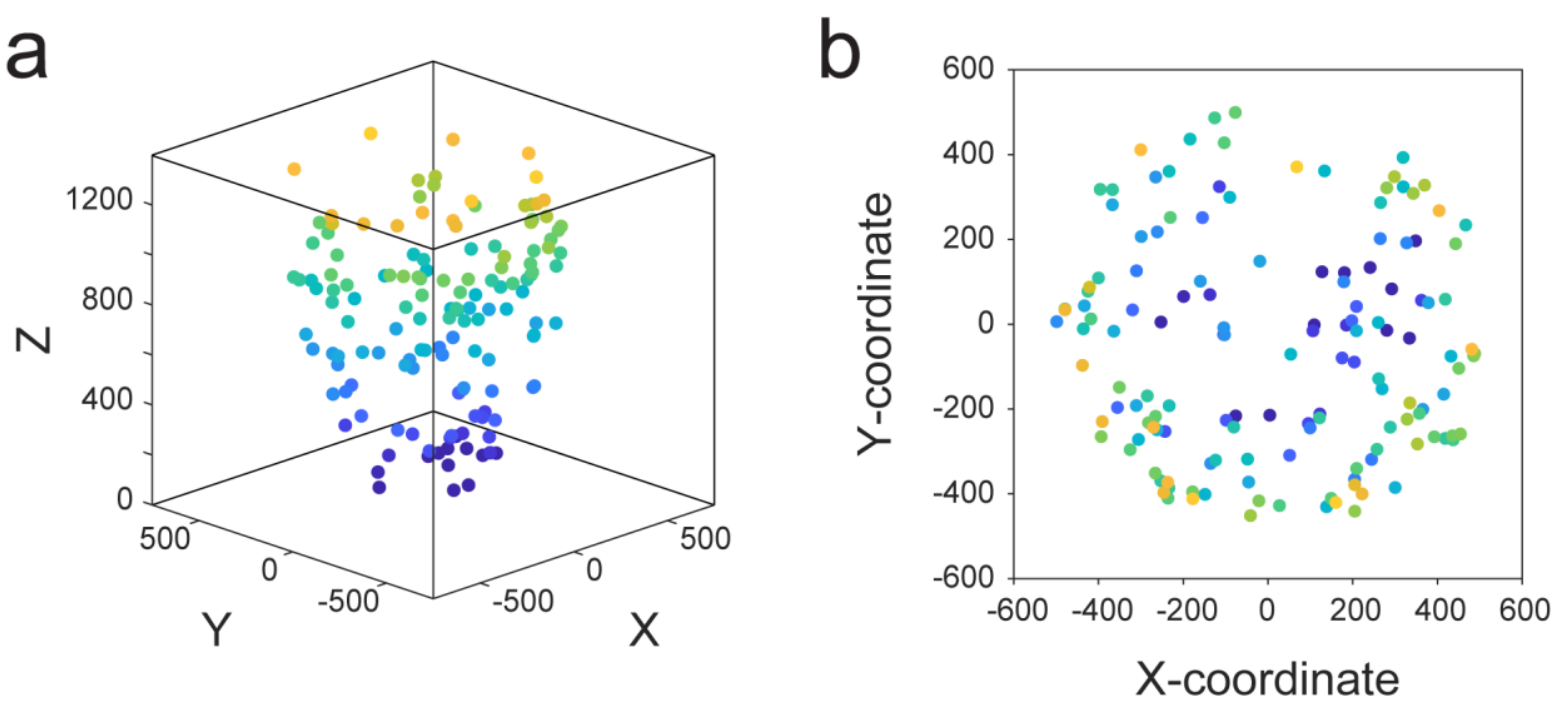

Figure S5. Targeting moiety positions in a 3D DNA-PAINT measurement. (a) 3D visualization of the targeting moiety positions. The color is an indication for the height. (b) $2 \mathrm{D}$ projection of the targeting moiety positions visualized in panel a. At the edge of the position cloud, the $z$-coordinates of the positions are larger compared to the positions in the middle of the cloud. The color indicates the z-coordinates, equal to panel a. 


\section{Supernatant assay}

A supernatant assay with Atto655-biotin was performed to determine the mean binding capacity per particle. Figure S6 shows the experimental results of the supernatant assay from which the saturation point was obtained as visualized in Figure 2a and Figure S3a. Figure S6a shows the measured fluorescence intensity of the supernatant as a function of the initial Atto655-biotin concentration. For the calibration curve where no NeutrAvidin-coated particles were incubated (blue dots) a linear dependency was observed (blue dashed line). When the Atto655-biotin solution was incubated with the NeutrAvidin-coated particles the dependency was no longer linear, since Atto655-biotin can bind to the NeutrAvidin complexes on the particles. By calculating the remaining Atto655-biotin concentration in the supernatant using the measured fluorescence intensity of the supernatant and the calibration curve, the binding capacity could be calculated. In Figure S6b, the amount of protein per particle and the binding capacity of a particle is given for two samples (each measured twice). From an absorbance measurement at $280 \mathrm{~nm}$, the amount of absorbed protein on the particle surface was determined to be $(2.6 \pm 0.4) \cdot 10^{5}$ (mean \pm SEM, two samples each measured twice). A binding capacity of (4.0 \pm 0.3$)$. $10^{5}$ (mean \pm fitting errors, two samples) Atto655-biotin molecules per particle follows from panel a. This results in $(2.0 \pm 0.2) \cdot 10^{5}$ targeting moieties per hemisphere which was observed in qPAINT experiments. The parameter $n$ indicates the number of accessible biotin-binding sites on the NeutrAvidin molecules, determined by dividing the total binding capacity by the number of protein complexes. This parameter was found to be $n=1.5 \pm 0.3$ Atto655-biotin molecules per NeutrAvidin complex.

a

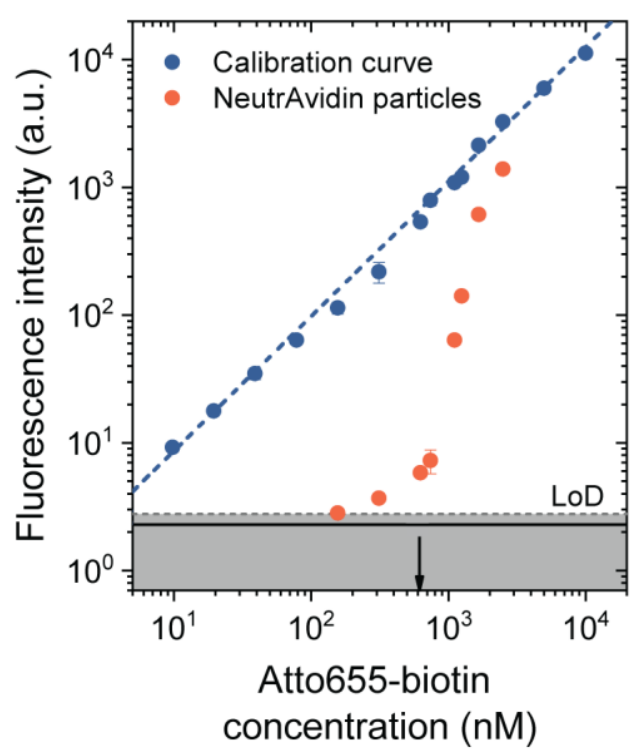

b

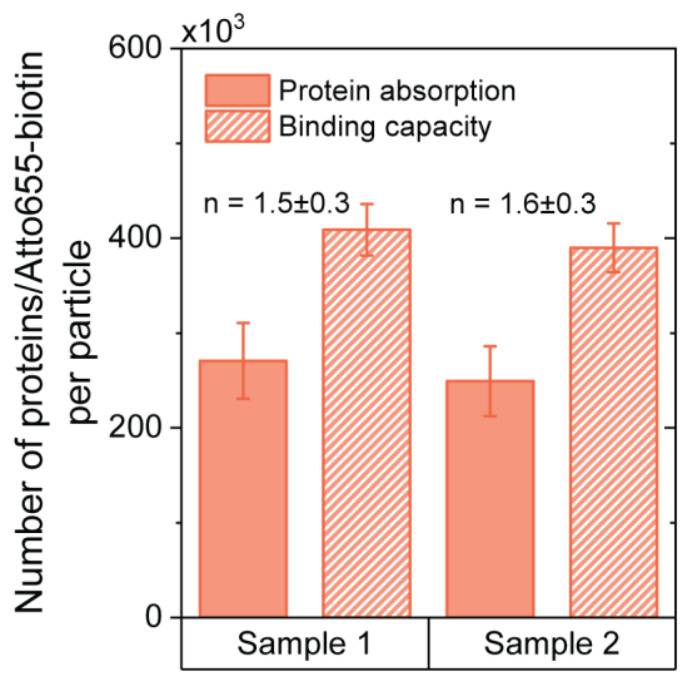

Figure S6. Supernatant assay to determine the binding capacity of NeutrAvidin-coated particles. (a) Supernatant assay with Atto655-biotin to determine the mean binding capacity per particle. The calibration curve (blue dots) shows a linear dependency (blue dashed line) between the measured fluorescence intensity and the Atto655-biotin concentration. When NeutrAvidin particles were added to an Atto655-biotin solution (red), no linear dependency could be observed, which shows that Atto655-biotin binds to NeutrAvidin. The black solid line indicates the background signal and the dashed grey line $\mu_{b}+3 \sigma_{b}$ (i.e. limit of detection, LoD) where $\mu_{b}$ is the background signal and $\sigma_{b}$ the standard deviation of the background signal. The arrow on the $x$-axis indicates the binding capacity in the experiment. (b) The protein absorption using absorption measurements was found to be $(2.7 \pm 0.4) \cdot 10^{5}$ and $(2.5 \pm 0.4) \cdot 10^{5}$ (mean \pm SEM) NeutrAvidin complexes per particle for sample 1 and sample 2 respectively, and the binding capacity that results from panel a was found to be $(4.1 \pm 0.3) \cdot 10^{5}$ and $(3.9 \pm 0.3) \cdot 10^{5}$ Atto655biotin molecules per particle (mean \pm fitting errors) for sample 1 and sample 2 respectively. The mean number of Atto655-biotin molecules per NeutrAvidin complex was found to be $1.5 \pm 0.3$ and $1.6 \pm 0.3$ for sample 1 and sample 2 respectively.

Based on the supernatant assay, a saturation point is expected for ssDNA concentrations higher than $6.3 \mu \mathrm{M}$. In the qPAINT data (see Figure $2 \mathrm{a}$ ), no saturation point was found. This absence of a saturation point in the qPAINT measurement is probably caused by two differences in experimental conditions between qPAINT and supernatant assay experiments. Firstly, the supernatant assay uses an excess of Atto655-biotin to quantify the saturation point which precludes depletion of Atto655-biotin. The association of Atto655-biotin to the particle is therefore faster compared to the association of ssDNA to 
the particle in the qPAINT experiment. Furthermore, Atto655-biotin is a smaller and a less charged molecule compared to SsDNA, causing less steric hindrance and charge repulsion on the particle surface respectively. These differences both cause a slower association of ssDNA to NeutrAvidin and thus a higher saturation point in qPAINT experiments compared to the supernatant assay.

Supernatant assay. $5 \mu \mathrm{L}$ NeutrAvidin-coated silica particles (1 wt.-\%) were added to $195 \mu \mathrm{L}$ PBS (130 $\mathrm{mM} \mathrm{NaCl}, 7 \mathrm{mM} \mathrm{Na}_{2} \mathrm{HPO}_{4}, 3 \mathrm{mM} \mathrm{NaH}_{2} \mathrm{PO}_{4}$ at pH 7.4). The particles were centrifuged at 14,500 RPM for 5 minutes using a tabletop spinner (Eppendorf MiniSpin) to clear the supernatant. The supernatant was carefully removed and discarded. $100 \mu \mathrm{L}$ Atto655-biotin (Sigma-Aldrich, $\geq 95.0 \%$ ) in PBS in the required concentration was added to the particles and vortexed to redisperse the particles. The particles were incubated with the Atto655-biotin for 3 hours on a rotating fin. Again, the particles were centrifuged at 14,500 RPM for 15 minutes to clear the supernatant. The supernatant was separated from the particles and the fluorescence intensity was measured with a plate reader (Fluoroskan Ascent) using a 384 well plate (Corning). 


\section{Size dispersion of silica particles}

In Figure S7, the size dispersion of silica particles was determined by scanning electron microscopy (SEM). A circle was fitted through each particle outline. The area of this circle was used to calculate the particle diameter and was found to be $0.97 \pm 0.04 \mu \mathrm{m}$ (mean $\pm \mathrm{SD}$ ). The size dispersion was quantified with the coefficient of variation $(C V)$ of the particle diameter and was found to be $C V_{\text {size }}=$ $3.9 \pm 0.5 \%$.
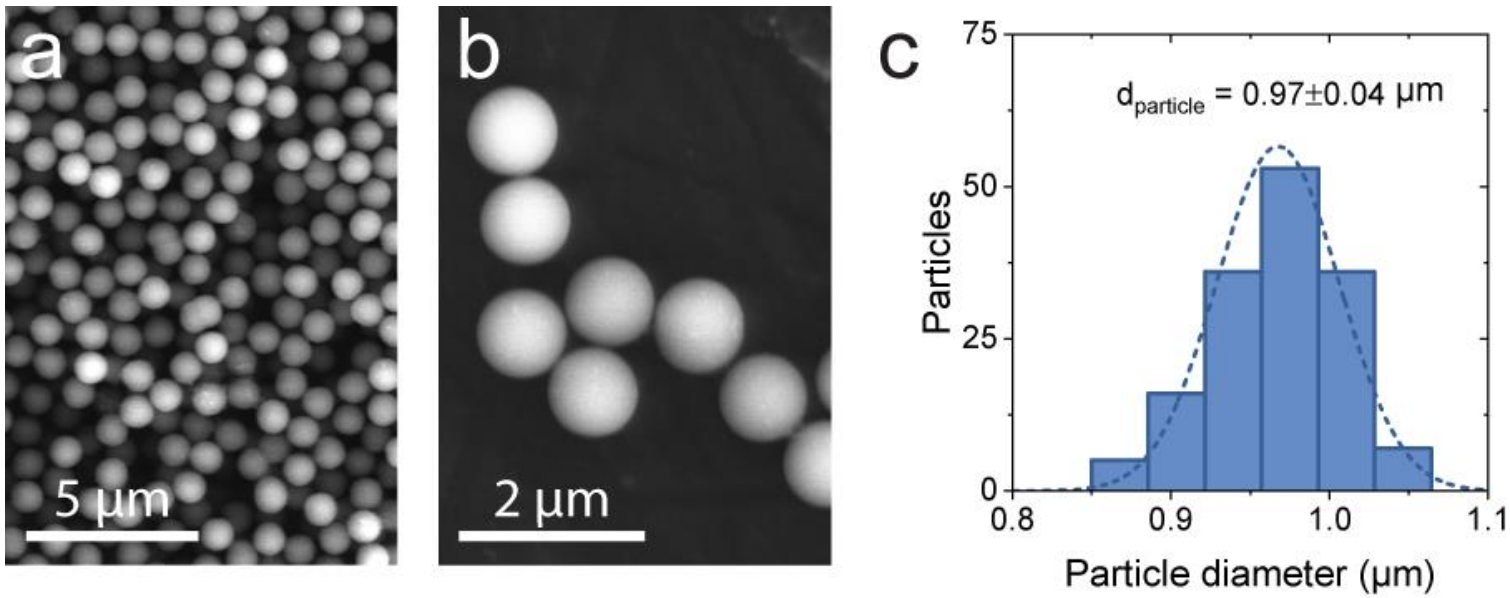

Figure S7. Size dispersion quantification of silica particles using scanning electron microscopy (SEM). (a) Overview SEM image with multiple silica particles. Scale bar indicates $5 \mu \mathrm{m}$. (b) Magnified SEM image of individual particles. Scale bar indicates $2 \mu \mathrm{m}$. (c) Histogram of the measured particle diameter. The mean diameter was found to be $0.97 \pm 0.04 \mu \mathrm{m}$ (mean \pm SD) which results in a coefficient of variation of $3.9 \pm 0.5 \%$ (mean \pm fitting error).

The size dispersion of silica particles is also estimated from DNA-PAINT images (see Figure S8). In Figure S8a, all DNA-PAINT localizations are visualized for a single field of view in a DNA-PAINT experiment where the particles are clearly visible as high-density localization clouds. The area of each localization cloud was determined by a convex hull, from which the diameter was calculated, which was found to be $1.09 \pm 0.05 \mu \mathrm{m}$ (mean $\pm \mathrm{SD}$ ). A $C V_{\text {size }}$ of $4.9 \pm 0.7 \%$ was calculated, which largely matches with the results presented in Figure S7c. The slightly larger diameter can be explained by the additional NeutrAvidin layer, the localization imprecision in DNA-PAINT measurements, and possible inclusion of nonspecific events close to the particle.
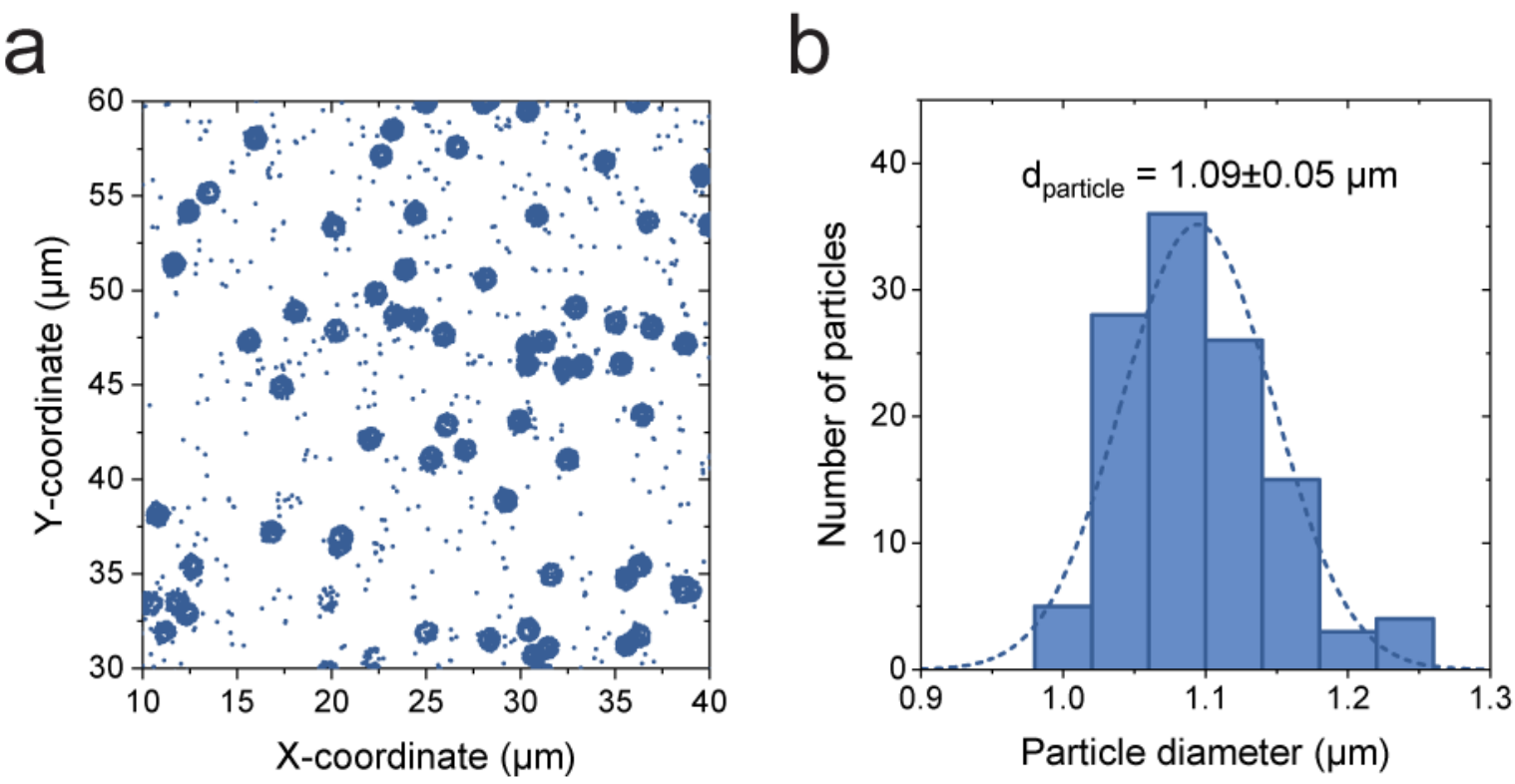

Figure S8. Size dispersion quantification of silica particles using DNA-PAINT localizations. (a) $x$ - and y-coordinates of all DNA-PAINT localizations in a single measurement. (b) The mean diameter of the localization cloud is assumed to represent the particle diameter $d_{\text {particle }}=1.09 \pm 0.05 \mu \mathrm{m}$ (mean \pm SD) which results in a coefficient of variation of $4.9 \pm 0.7 \%$ (mean \pm fitting error). 


\section{Clark-Evans test}

The Clark-Evans (CE) test is used to compare the mean observed nearest-neighbor (NN) distance between two targeting moiety positions to the expected mean NN-distance based on the targeting moiety density. Based on this test, the targeting moiety positions can be significantly dispersed, where the mean NN-distance is larger than expected (i.e. ordered positions), significantly clustered, where the mean NN-distance is shorter than expected, or randomly positioned, where the mean NN-distance is comparable to what is expected under the complete spatial randomness (CSR) hypothesis. The CSR hypothesis was tested using a standardized sample mean $z_{m} \sim N(0,1)$ which is a measure for the degree of clustering (negative $z_{m}$ ) or dispersion (positive $z_{m}$ ):

$$
z_{m}=\frac{\bar{d}_{m}-\widehat{\mu}}{\widehat{\sigma}}
$$

with $\bar{d}_{m}=\frac{1}{m} \sum_{i=1}^{m} D_{i}$ being the observed mean NN-distance of $m$ targeting moiety positions, $\hat{\mu}=\frac{1}{2 \sqrt{\lambda}}$ the expected mean NN-distance based on a targeting moiety density $\lambda$ and $\hat{\sigma}^{2}=\frac{4-\pi}{m(4 \lambda \pi)}$ the expected variance of the NN-distance. In order to use this method to accept or reject the CSR hypothesis, three requirements have to be met.

1. The total number of DNA-PAINT localizations $N_{L}$ per particle should be high enough to accurately accept or reject the CSR hypothesis, i.e. the number of targeting moieties falsely classified as clustered (false positives) should be low.

2. The number of reflexive nearest-neighbors and edge effects should be minimized, to prevent including double nearest-neighbors and to prevent increased nearest-neighbor distances respectively.

3. The chance that a binder is observed multiple times should be minimized, i.e. mean number of localizations per binder $\lambda$ is low.

In Figure S9, the results of the optimization of the CE-method have been visualized, where the aforementioned requirements are considered. Random positions of targeting moieties were simulated on a particle hemisphere for a total of 50 particles with $N_{L}=150, m=30$ and $10 \%$ edge removal (see black arrows on $\mathrm{x}$-axes). For panels $\mathrm{a}-\mathrm{b}$, the number of targeting moiety locations per particle equals the number of localizations per particle, i.e. no stochastic binding of imager strand was simulated. However, for panel c, stochastic binding was included.

Figure S9a shows the dependency of $z_{m}$ on the number of localizations per particle $N_{L}$. The error with which $z_{m}$ can be calculated depends on $N_{L}$ due to statistics, since the error scales according to $\sigma \propto$ $N_{L}^{-0.5}$. In Figure S9b (top) the number of reflexive NNs is reduced by taking $10^{3}$ random subsamples of size $m$ from all localizations and calculate $z_{m}$ for all subsamples; the mean $z_{m}$ was reported as the $z_{m}$ value of that particular particle. By increasing the subsample size $m, z_{m}$ shows no change in its mean value while the variance increases, where the error of $z_{m}$ scales with $\sigma_{z_{m}} \propto \sqrt{m}$. However, since the estimated variance of the NN-distances scales with $\hat{\sigma} \propto m^{-0.5}$ (see Equation S3), a balance has to be found between reducing the effect of reflexive neighbors and a reasonable error of the estimated mean $\mathrm{NN}$-distance. In Figure S9b (bottom) the dependency of $z_{m}$ on the edge localizations removal is visualized. Here the $x$-axis resembles the percentage of the $z$-coordinate span which is removed from analysis. Using this approach, the highest z-coordinates (at the edge of the hemisphere) are removed from analysis. By including all localizations, the $z_{m}$ value increases since the mean NN-distance for localizations at the edge is larger, compared to localizations in the middle of the hemisphere. By removing $10 \%$ of the edge, no bias was observed in the calculated $z_{m}$ value. In conclusion, a number of localizations $N_{L}=150$ and subsample size $m=30$ yields approximately $0.1 \%$ false positives in rejecting the CSR hypothesis (one-tailed test with $\alpha=0.05$ ).

In Figure S9c the stochastic sampling of the targeting moieties was simulated for nonclustered targeting moieties (blue), and $25 \%$ clustered targeting moieties (red, equal to the $25 \%$ clustered simulation in Figure $3 \mathrm{~b}$ ), where the calculated $z_{m}$ value is given as a function of the mean number of localizations per targeting moiety $\lambda$. $\lambda$ can be tuned by the imager strand concentration and the duration of the measurement and was approximately equal for all ssDNA coverages given in Figure 3c. The simulations for no clustering show induced clustering by oversampling, since the targeting moieties could be observed more than once in a DNA-PAINT experiment. It was found that for $0.05 \geq \lambda \geq 0.15$ (shaded area) induced clustering is minimized, while the difference between no clustering and 
clustering is observable. Larger values for $\lambda$ would result in a percentage of targeting moieties that are imaged more than once of $>1 \%$.

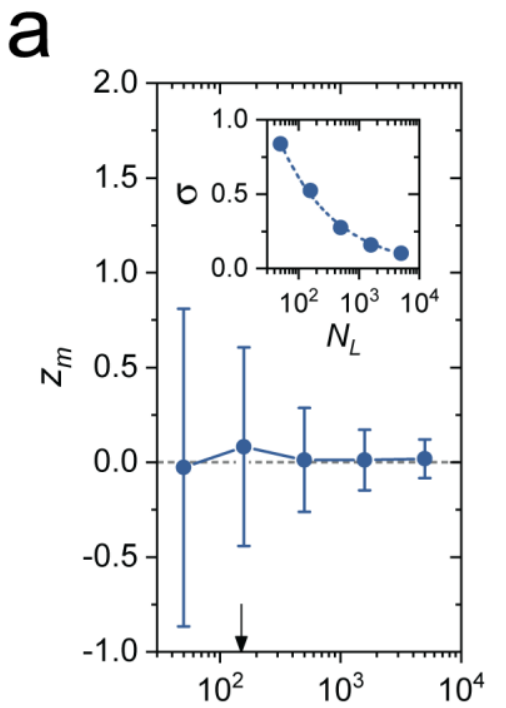

Number of localizations $N_{L}$ b

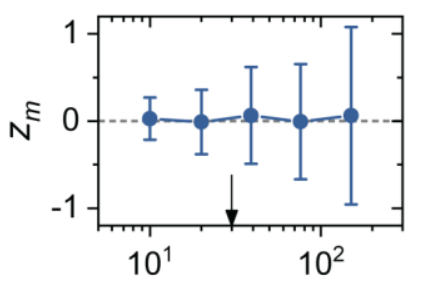

Subsample size $m$

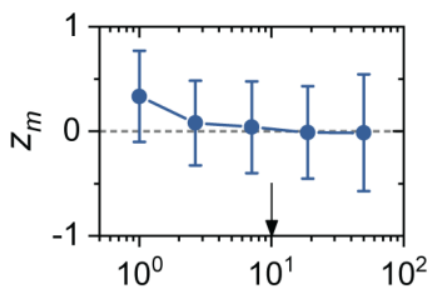

Edge removal (\%)
C

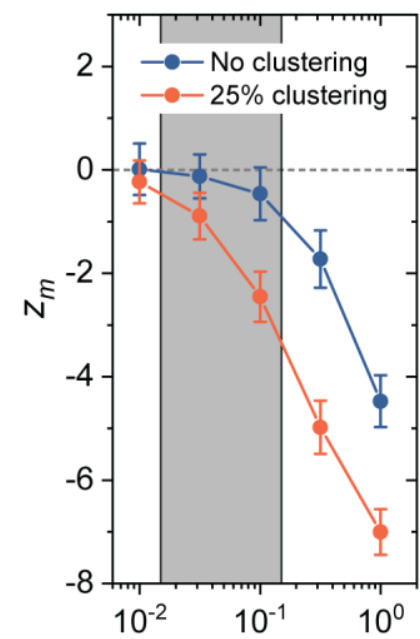

Mean number of localizations

per targeting moiety $\lambda$

Figure S9. Optimizing the CE-test to accept or reject the complete spatial randomness (CSR) hypothesis. (a) The dependency of the $z_{m}$ value and its error on the number of localizations per particle $N_{L} . N_{L}=150$ (black arrow) is chosen to accurately reject the CSR hypothesis (one-tailed test with $\alpha=0.05 \rightarrow \mathrm{FP} \sim 0.1 \%$ ). The following parameters were used: $m=30$, edge removal $=10 \%, R_{p}=500 \mathrm{~nm}$, and no Poisson sampling process. The error of $z_{m}$ is shown in the inset which scales with the number of localizations according to $\sigma \propto N_{L}^{-1 / 2}$ as would be expected with Poisson statistics. (b) Top: the effect of reflexive NNs. Including reflexive NNs causes an increase of the variance of the mean $z_{m}$ value. Bottom: dependency of $z_{m}$ on the edge localizations removal; including particle probe positions at the edge increases the mean $z_{m} \cdot m=150$ and edge removal $=10 \%$ (black arrows) were chosen required to accurately reject the CSR hypothesis (one-tailed test with $\alpha=0.05 \rightarrow \mathrm{FP} \sim 0.1 \%$ ). The following parameters were used: $N_{L}=150, m=30$ (bottom) or edge removal $=10 \%$ (top), $R_{p}=500 \mathrm{~nm}$, and no Poisson sampling process. (c) Stochastic sampling of the targeting moieties for nonclustered targeting moieties (blue), and $25 \%$ clustered targeting moieties (red, see Figure $3 \mathrm{~b}$ ), with $z_{m}$ as a function of the mean number of localizations per targeting moiety $\lambda$. Both curves show a decreasing $z_{m}$ for an increasing $\lambda$ due to oversampling and therefore induced clustering. The shaded area indicates the range in which the oversampling is minimized, while the difference between nonclustered and clustered targeting moieties could be distinguished. The following parameters were used: $N_{L}=150, m=30$, edge removal $=10 \%, R_{p}=500 \mathrm{~nm}$, and a Poisson sampling process. For all simulations each data point consists of 50 particles of which the means and standard deviations are visualized. 


\section{Scaling of intraparticle targeting moiety variability with interaction area}

The contribution of intraparticle variability due to clustered functionalization on a particle to the reactivity variability for a given interaction area can be defined by:

$$
\sigma_{\text {moiety }}^{2}=\sigma_{\text {intraparticle }}^{2}+\sigma_{\text {stochastic }}^{2} \rightarrow \sigma_{\text {intraparticle }}^{2}=\sigma_{\text {moiety }}^{2}-N_{\text {moiety }}
$$

with $\sigma_{\text {moiety }}$ the variation in the number of targeting moieties for a given interaction area, $\sigma_{\text {intra-particle }}$ the intraparticle variation for a given interaction area, $\sigma_{\text {stochastic }}$ the variability caused by stochastic functionalization of targeting moiety, and $N_{\text {moiety }}$ the mean number of targeting moieties in a given interaction area. All parameters are a function of the interaction area $a_{i}$.

In Figure S10a the variation in the number of targeting moieties is given as a function of $a_{i}$ using the simulations presented in Figure $3 \mathrm{~b}$. It was found that both the random, nonclustered particles (blue) and clustered particles (red) show $C V \propto a_{i}^{-0.5}$ (dashed blue and red lines). This relation changes at the outer limits (dashed grey lines) due to simulation artefacts. For small interaction areas, the distribution changes from a normal distribution to a Poisson distribution causing an underestimation of $\sigma_{\text {moiety }}$. For large interaction areas $\sigma_{\text {moiety }}$ approaches zero since a fixed number of targeting moieties was used for each simulated particle to exclude interparticle variation effects. Figure $\mathrm{S} 10 \mathrm{~b}$ shows $\sigma_{\text {intra-particle }}$ as a function of the interaction area using Equation S4. Similar to the variation in the number of targeting moieties, the intraparticle variation scales with $C V \propto a_{i}^{-0.5}$ (dashed red line).
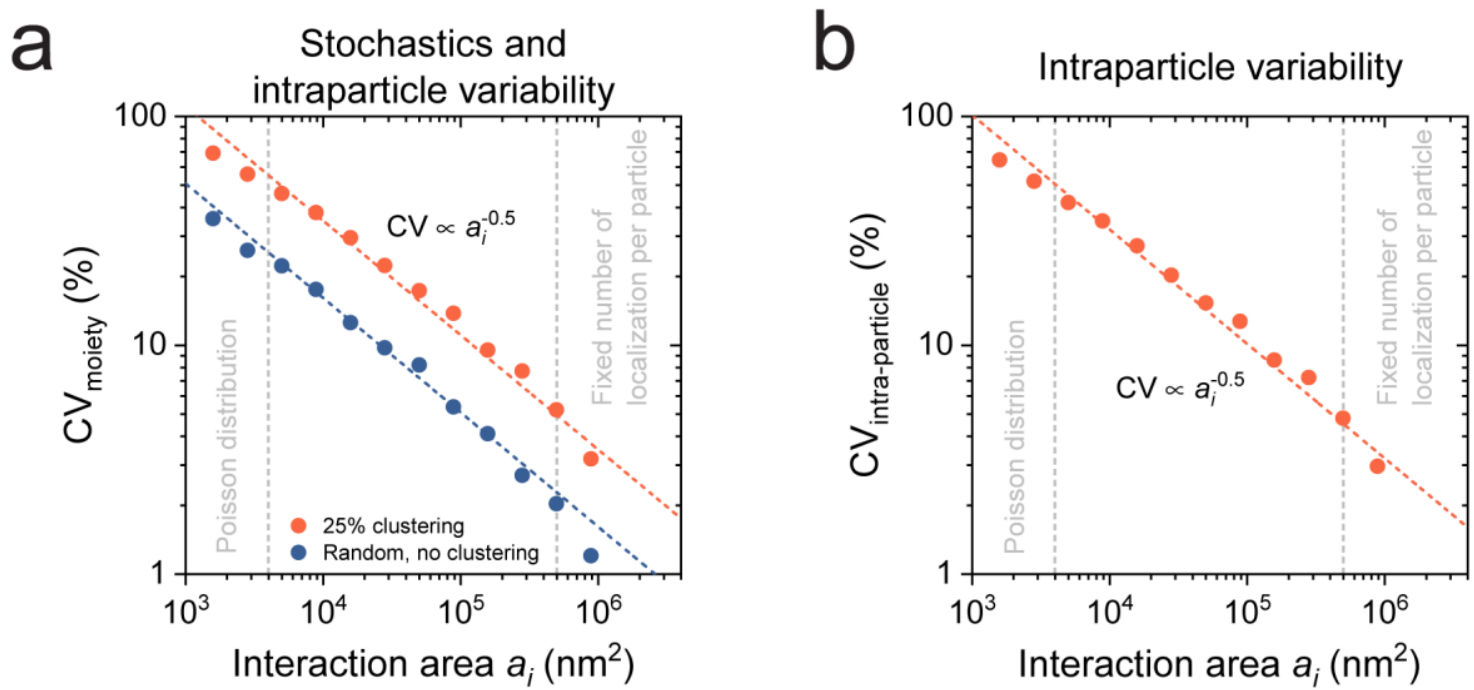

Figure S10. Scaling of the intraparticle variability with the interaction area due to clustered targeting moiety functionalization. (a) The variation in the number of targeting moieties as a function of the interaction area, where both stochastic and the intraparticle variability are taken into account, for particles with a random placement of targeting moieties (blue) and a superposition of $25 \%$ clustered and $75 \%$ random placement of targeting moieties (red). The dashed blue and red lines indicate $C V \propto a_{i}^{-0.5}$. The grey dashed lines indicate the area between which no simulation artefacts occur. (b) The variation in the number of targeting moieties as a function of the interaction area, where only the intraparticle variability was considered using Equation S4. The dashed red line indicates $C V \propto a_{i}^{-0.5}$. 


\section{Correlation between inter- and intraparticle targeting moiety variabilities}

The correlation between the interparticle variability quantified by qPAINT experiments, and the intraparticle variability quantified by DNA-PAINT experiments, is shown in Figure S11. Here, the calculated $z_{m}$ value is indicated on the $\mathrm{x}$-axis, and the number of targeting moieties per particle measured using qPAINT on the $y$-axis. Each dot represents a single particle, and the red cross the mean value of the $z_{m}$ value and the number of targeting moieties per particle. This Figure shows that (1) there is no correlation between intraparticle variability and number of targeting moieties on a single particle, and (2) the spread (i.e. the variation) in the distribution of the number of targeting moieties per particle does not depend on the $z_{m}$ value. Both observations are in agreement with the fact that the intrinsic length scales of inter- and intraparticle heterogeneity are very different, so that these heterogeneities can be treated as independent terms.

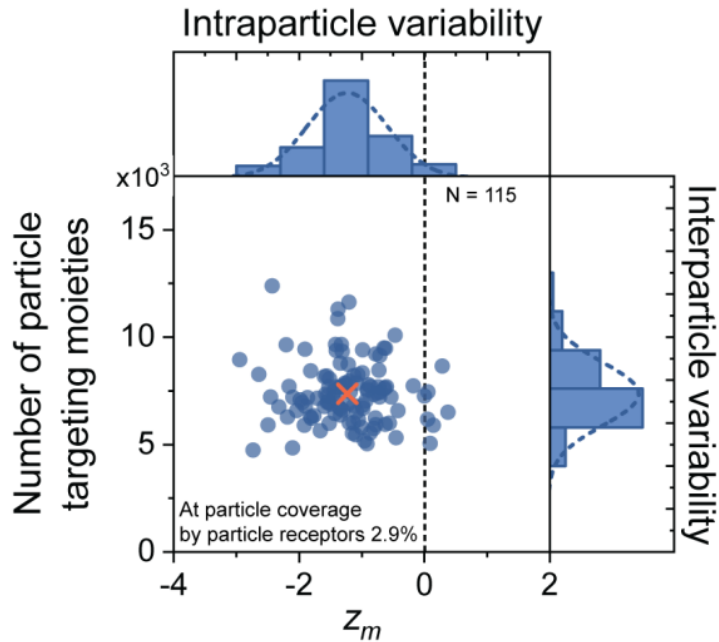

Figure S11. Correlation between inter- and intraparticle variabilities. On the x-axis, $z_{m}$ is given (see Figure 3c), and on the $y$-axis the number of targeting moieties (see Figure 2a) for a particle coverage of $2.9 \%$. Each blue dot represents a single particle for which the number of targeting moieties was determined using qPAINT and for which $z_{m}$ was calculated using DNA-PAINT data. The red cross indicates the mean number of targeting moieties and mean $z_{m}$. The dashed blue lines indicate a normal distribution. 


\section{Biosensing by particle mobility}

\subsection{Biosensing by particle mobility assay principle}

In this paper the concept and consequences of superpositional heterogeneity for the variability in reactivity of biofunctionalized particles, are illustrated using biosensing by particle mobility (BPM), a biosensing method with both single-particle and single-molecule resolution..$^{5-7}$ The molecular design and measurement principle are sketched in Figure S12, illustrated with a sandwich assay format. Figure $\mathrm{S} 12 \mathrm{a}$ shows a particle that is tethered to a substrate by a molecular nanoswitch system comprising three functional components: ${ }^{5}$ (1) a double-stranded DNA (dsDNA) stem which tethers the particle to the substrate, (2) a ssDNA targeting moiety coupled to the stem, and (3) multiple ssDNA targeting moieties coupled to the particle surface. Figure S12b illustrates the sensing functionality of the BPM system. The stem targeting moiety can transiently bind to target molecules captured from solution by the targeting moieties on the particle. The transient binding affects the mobility of the particle, because an unbound particle has a larger in-plane motional freedom than a bound particle. Two mobility time traces are sketched in Figure S12c, at a high (left) and low (right) target concentration. The switching frequency of the particle depends on the target concentration, because the unbound state lifetime of a particle decreases when the number of captured target molecules increases.
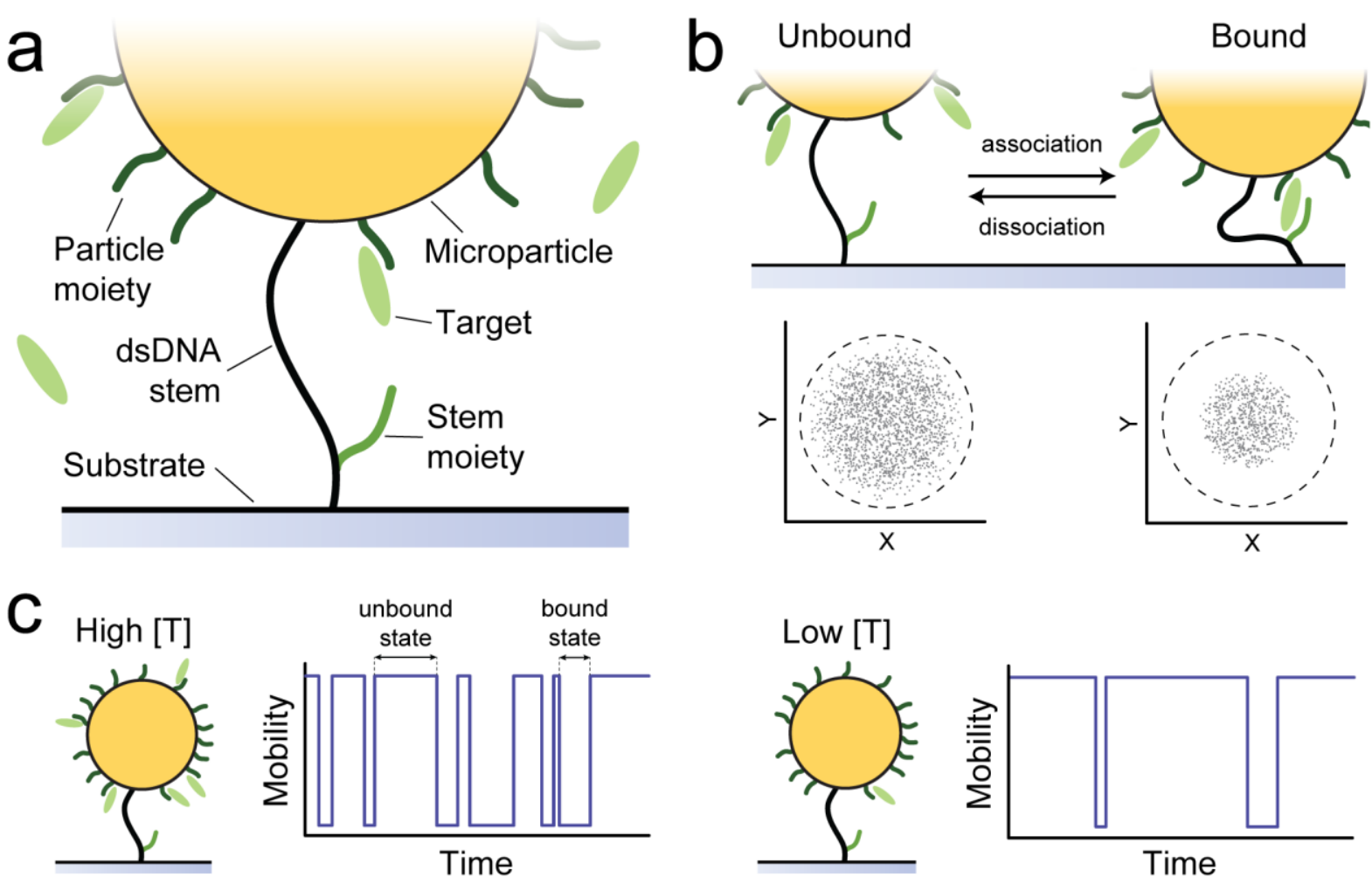

Figure S12. Molecular design and measurement principle of biosensing by particle mobility (BPM) using a single targeting moiety on the stem. (a) Micrometer-sized particles (yellow) are tethered to a substrate using a dsDNA stem (black). The particle is functionalized with targeting moieties (dark green) and a single stem targeting moiety (green). Both targeting moiety types can bind reversibly to single target molecules (light green) present in solution. (b) Target molecules binding to the targeting moieties on the particle and subsequently the targeting moiety on the stem cause the particle to exhibit either of two concentric Brownian motion patterns, i.e. the projection of the center if the particle onto the xy-plane, corresponding to the unbound (high mobility) and bound state (low mobility). (c) Digital binding and unbinding events are identified by following the mobility of the particles over time. The time between two events corresponds to either the unbound state lifetime, or the bound state lifetime. For a high or low target concentration in solution, the microparticle shows a high or a low switching frequency respectively.

\subsection{Interaction area}

Here the interaction area $a_{i}$ is calculated for two BPM designs, namely the BPM sensor with single stem targeting moiety ${ }^{5}$ and the BPM sensor having the substrate coated with multiple targeting 
moieties, ${ }^{6}$ see Figure $S 13$. The interaction area can be calculated using the area formula for a spherical cap:

$$
a_{i}=2 \pi R_{p}^{2} \cdot(1-\cos \alpha)
$$

with $R_{p}$ being the particle radius, and $\alpha$ the angle between the rays from the center of the sphere to the apex of the cap and the edge of the cap. The angle $\alpha$ can be calculated using $d=R_{p} \alpha$ where $d$ is the great-circle distance from the center to the edge of the cap of the interaction area.

Figure $\mathrm{S} 13$ shows the BPM geometries for a small ${ }^{5}$ and large interaction area. ${ }^{6}$ The interaction areas are $a_{i}=6.4 \cdot 10^{3} \mathrm{~nm}^{2}$ (i.e. $\sim 0.2 \%$ of the total particle area, see Figure S13a) and $a_{i}=6.1 \cdot 10^{4} \mathrm{~nm}^{2}$ $(\sim 2 \%$ of the total particle area, see Figure S13b), respectively. The BPM design with a small interaction area exhibits variabilities dominated by stochastic and intraparticle heterogeneity. The BPM design with a large interaction area has variability due to all three components (stochastic, intra particle, and inter particle).
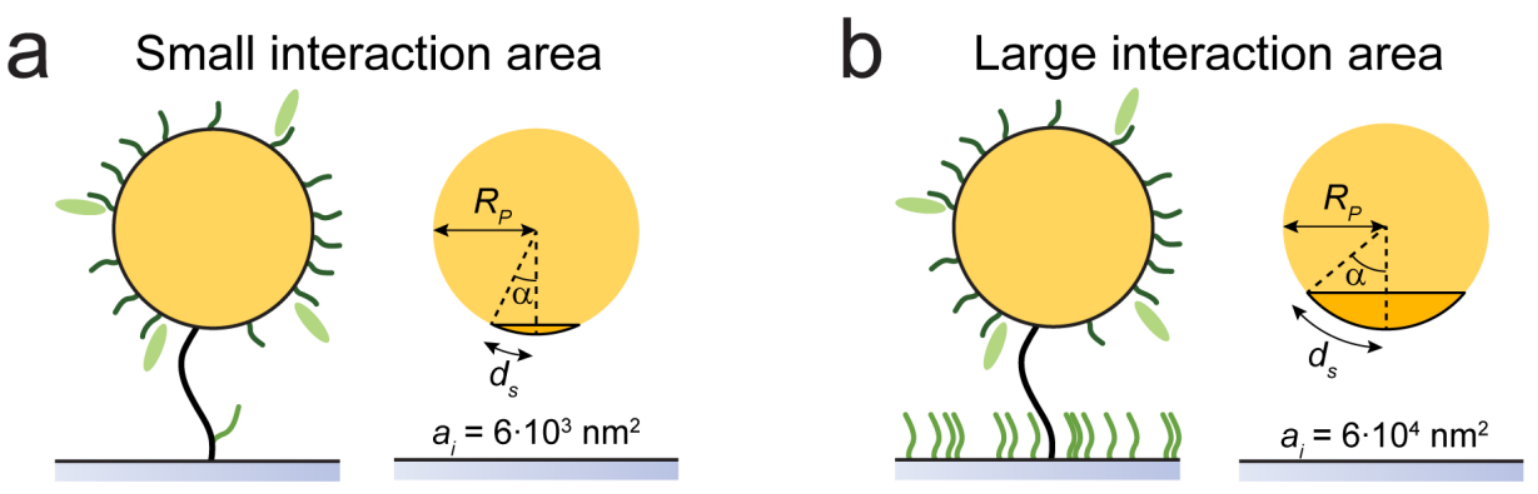

Figure S13. Schematic visualizations of the interaction area for two BPM system designs. (a) Schematic visualization of the BPM design presented in Lubken et al. ${ }^{5}$ The characteristic length of the interaction area $d$ was estimated to be $45 \mathrm{~nm}$ which results in $\alpha \sim 5^{\circ}$. (b) Schematic visualization of the BPM design presented in Yan et al. ${ }^{6}$ The characteristic length of the interaction area $d$ was estimated to be $140 \mathrm{~nm}$ which results in $\alpha \sim 16^{\circ}$. 


\section{REFERENCES}

(1) Jungmann, R., Avendaño, M.S., Dai, M., Woehrstein, J.B., Agasti, S.S., Feiger, Z., Rodal, A. \& Yin, P. Quantitative Super-Resolution Imaging with qPAINT. Nature Methods 13, 439-442 (2016).

(2) Delcanale, P., Miret-Ontiveros, B., Arista-Romero, M., Pujals, S. \& Albertazzi, L. Nanoscale Mapping Functional Sites on Nanoparticles by Points Accumulation for Imaging in Nanoscale Topography (PAINT). ACS Nano 12, 7629-7637 (2018).

(3) Horáček, M., Engels, D.J. \& Zijlstra, P. Dynamic Single-Molecule Counting for the Quantification and Optimization of Nanoparticle Functionalization Protocols. Nanoscale 12, 4128-4136 (2020).

(4) Jungmann, R., Steinhauer, C., Scheible, M., Kuzyk, A., Tinnefeld, P. \& Simmel, F.C. SingleMolecule Kinetics and Super-Resolution Microscopy by Fluorescence Imaging of Transient Binding on DNA Origami. Nano Letters 10, 4756-4761 (2010).

(5) Lubken, R.M., de Jong, A.M. \& Prins, M.W.J. Multiplexed Continuous Biosensing by SingleMolecule Encoded Nanoswitches. Nano Letters 20, 2296-2302 (2020).

(6) Yan, J., van Smeden, L., Merkx, M. Zijlstra, P. \& Prins, M.W.J. Continuous Small-Molecule Monitoring with a Digital Single Particle Switch. ACS Sensors 5, 1168-1176 (2020).

(7) Visser, E.W.A., Yan, J., van IJzendoorn, L.J., \& Prins, M.W.J. Continuous Biomarker Monitoring by Particle Mobility Sensing with Single Molecule Resolution. Nature Communications 9, 2541 (2018). 